\title{
Dose-response and dose-toxicity relationships for yttrium-90 glass radioembolization in patients with colorectal cancer liver metastases
}

Ahmed A. Alsultan*, Caren van Roekel, Maarten W. Barentsz, Maarten L.J. Smits, Britt Kunnen, Miriam Koopman, Arthur J.A.T. Braat, Rutger C.G. Bruijnen, Bart de Keizer, Marnix G.E.H. Lam.

${ }^{*}$ Corresponding author

Affiliation of all authors: Division of Imaging and Oncology, University Medical Center Utrecht, Utrecht University.

Corresponding author:

Ahmed A. Alsultan

$+31887574675$

A.A.N.Alsultan@umcutrecht.nl

University Medical Center Utrecht

Heidelberglaan 100

3584CX Utrecht

The Netherlands

ORCID:0000-0003-4236-1376

WC: 5000

Short running title: Dose-effect Y-90 radioembolization 


\section{ABSTRACT}

Purpose Radioembolization based on personalized treatment planning requires established doseresponse and dose-toxicity relationships. The aim of this study was to investigate dose-response and dose-toxicity relationships in patients with colorectal liver metastases (CRLM) treated with glass yttrium-90 $\left({ }^{90} \mathrm{Y}\right)$-microspheres. Methods All CRLM patients treated with glass ${ }^{90} \mathrm{Y}$-microspheres in our institution were retrospectively analyzed. The tumor-absorbed dose was calculated for each measurable metastasis (i.e., ${ }^{18} \mathrm{~F}-\mathrm{FDG}-$ positive and $>5 \mathrm{~mL}$ tumor volume) on post-treatment ${ }^{90} \mathrm{Y}$-PET. Metabolic tumor response was determined on ${ }^{18} \mathrm{~F}-\mathrm{FDG}-\mathrm{PET} / \mathrm{CT}$ by measuring the total lesion glycolysis at baseline and at three months post-treatment. The relationship between tumor-absorbed dose and metabolic response was determined on a per lesion and per patient basis using a linear mixed-effects regression model. Clinical and laboratory toxicity were correlated with healthy liver-absorbed dose. Results Thirty-one patients were included. The median tumor-absorbed dose of 85 measurable metastases was 133 Gy (range 20-1001 Gy). Per response category this was 196 Gy for complete response (CR), 177 Gy for partial response (PR), 72 Gy for stable disease, and 95 Gy for progressive disease (PD). A significant dose-response relationship was found on a tumor level with a significantly higher tumor-absorbed dose in metastases with CR (+94\%) and PR (+74\%) compared to metastases with PD, $p<0.001$. A similar relationship was found on a patient level, with PR having a higher tumor-absorbed dose compared to PD $(+58 \%, p=0.044)$. A tumor-absorbed dose of >139 Gy predicted three-month metabolic response with the greatest accuracy (89\% specificity, $77 \%$ sensitivity), while a tumor-absorbed dose of $>189$ Gy predicted response with $97 \%$ specificity and $45 \%$ sensitivity. The median healthy liver-absorbed dose was 63 Gy (range: 24-113 Gy). Toxicity was mostly limited to grade $1-2$, with one case of radioembolization-induced liver disease who received the highest healthy liver-absorbed dose. A positive trend was seen for most laboratory parameters in our dose-toxicity analysis. Conclusion A significant relation was observed between dose and response in CRLM patients treated with glass ${ }^{90} \mathrm{Y}$-radioembolization.

Key words: Radioembolization, dose-response relationship, ${ }^{90} \mathrm{Y}$ PET, colorectal liver metastases, total lesion glycolysis. 


\section{INTRODUCTION}

Radioembolization is an established treatment option for patients with unresectable primary and secondary liver tumors (1). Microspheres containing yttrium-90 $\left({ }^{90} \mathrm{Y}\right)$ or holmium-166 $\left({ }^{166} \mathrm{Ho}\right)$ are injected intra-arterially to deliver a high radiation dose to the tumors. In hepatocellular carcinoma (HCC) the treatment effect was shown to be dependent on the tumor-absorbed dose, with higher doses achieving better response $(2,3)$. However, increasing the tumor-absorbed dose by administering higher activities also increases the healthy liver-absorbed dose. The relative distribution of microspheres between healthy liver tissue and tumor tissue, which varies greatly between different patients and tumor types, can be predicted by performing a simulation procedure prior to the treatment itself. This distribution can be used to perform compartment-model dose-planning, allowing for high tumor-absorbed doses while staying within the safety limits of the healthy liver-absorbed dose. This is especially important in patients with colorectal liver metastases (CRLM) who tend to have less favorable tumor-to-healthy liver distributions compared with patients with other tumor types. In one study, CRLM patients had a mean tumor-to-nontumor uptake ratio of 1.7 compared to 7.2 in $\mathrm{HCC}$ (4). Furthermore, CRLM patients may have received hepatotoxic systemic treatment, hepatic surgery, or local ablative procedures, limiting the tolerable healthy liver-absorbed dose.

A strong dose-response relationship in CRLM patients was found for radioembolization using resin ${ }^{90} \mathrm{Y}$-microspheres and ${ }^{166} \mathrm{Ho}$-microspheres but has not been demonstrated for glass ${ }^{90} \mathrm{Y}$-microspheres $(5,6)$. The dose-response relationship for glass ${ }^{90} Y$-microspheres is not equal to the relationship for resin

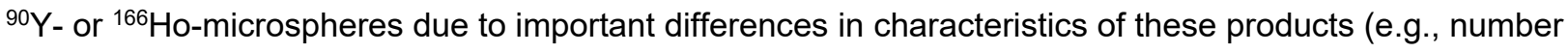
of microspheres, distribution, specific activity, radioisotope, density).

The aim of this study was to investigate dose-response and dose-toxicity relationships of glass ${ }^{90} \mathrm{Y}$-microspheres radioembolization in CRLM patients. 


\section{MATERIALS AND METHODS}

\section{Study Design and Patient Selection}

All CRLM patients treated with glass ${ }^{90} \mathrm{Y}$-microspheres, between January 2012 and December 2019 at our institute, were screened for inclusion. This study was approved by our ethical research committee and the need for informed consent was waived.

Reasons for exclusion for this study were: no ${ }^{18} \mathrm{~F}-\mathrm{FDG}-\mathrm{PET}$ performed or performed more than 10 weeks prior to treatment, previous whole-liver radioembolization, poor image registration, and no posttreatment ${ }^{90}$ Y-PET. To qualify for the dose-response evaluation, patients were required to have had followup ${ }^{18} \mathrm{~F}-\mathrm{FDG}-\mathrm{PET} / \mathrm{CT}$ performed within 2-4 months after treatment and FDG positive tumors $>5 \mathrm{~mL}$ at baseline. For the dose-toxicity evaluation, patients who received sequential whole-liver treatments (i.e., right, and left lobes treated separately) $<3$ months were excluded due to time-interval bias and inaccuracy of healthy liver-absorbed dose measurement.

\section{Treatment Procedures}

Candidates for ${ }^{90} \mathrm{Y}$-radioembolization treatment underwent work-up with ${ }^{18} \mathrm{~F}-\mathrm{FDG}$-PET/CT and multiphasic CT of the liver and were discussed in a multidisciplinary tumor board. All patients had to be in acceptable clinical condition (World Health Organization performance score 0-2) and have adequate organ function. One to two weeks before treatment, eligible patients underwent preparatory angiography, in which a surrogate dose $( \pm 150 \mathrm{MBq})$ of technetium-99m macro-aggregated albumin ( ${ }^{99 \mathrm{~m} T \mathrm{Tc}-\mathrm{MAA}) \text { was }}$ administered to simulate the intra- and extra-hepatic distribution of microspheres. This distribution was then visualized using ${ }^{99 m}$ Tc-MAA-SPECT/CT imaging.

Patients were treated palliatively in lobar and (sequential) whole-liver fashion, or as a bridge to resection as radiation lobectomy or segmentectomy. Treatment planning was performed using onecompartment modeling according to the medical internal radiation dose (MIRD) method, aiming for an average absorbed dose of 80-150 Gy (>200 Gy in radiation segmentectomy) in the treated volume (7). Post-treatment distribution was assessed using ${ }^{90} \mathrm{Y}-\mathrm{PET} / \mathrm{CT}$ imaging the morning after treatment. ${ }^{18} \mathrm{~F}-$ FDG-PET/CT and multiphasic-CT of the liver were acquired at three months after treatment for response assessment. 


\section{Dose-response Evaluation}

Tumor-absorbed dose and metabolic tumor response were assessed using ${ }^{90} \mathrm{Y}-\mathrm{PET} / \mathrm{CT}$ and ${ }^{18} \mathrm{~F}-$ FDG-PET/CT, respectively. This assessment was performed on a per-tumor basis and on a per-patient basis (using a weighted average of all measured tumors within a liver). All tumor delineations and image registrations were performed using Simplicit ${ }^{90} Y \circledR$ software (Mirada Medical Ltd.). Tumor volumes of interest (VOIs) were defined as previously reported $(5,8)$. In short, tumors were delineated using baseline ${ }^{18}$ F-FDG-PET/CT using a threshold for metabolic activity concentration as defined by PERCIST (9) (Fig. 1). A volume restriction of $\geq 5 \mathrm{~mL}$ was applied to solitary tumors. Merged tumors on follow-up were separated visually using contrast-enhanced CT imaging, if this could not be achieved, the merged tumors would be considered as one tumor at baseline for calculation of metabolic activity and absorbed dose. Total lesion glycolysis (TLG) was calculated for each lesion at baseline and at three-months follow-up to determine the metabolic response. Metabolic tumor response were categorized as follows: changes in metabolic activity of $-100 \%$ for complete metabolic response (CR), $-45 \%$ to $-99 \%$ for partial metabolic response (PR), $+75 \%$ to $-44 \%$ for stable disease (StD), and $+75 \%$ for progressive metabolic disease (PD). The categories were subsequently grouped into objective response $(C R+P R)$ and non-response (StD+PD). Occurrence of new lesions after radioembolization was reported, but not regarded as progressive disease in the analysis, as the goal was to demonstrate a dose-response relationship.

${ }^{18} \mathrm{~F}-\mathrm{FDG}-\mathrm{PET}$ images were co-registered to the ${ }^{90} \mathrm{Y}-\mathrm{PET}$, using the low-dose CT-scans. To improve measurement accuracy, the ${ }^{90}$ Y-PET dose-map was used to register each tumor VOI individually. Only rigid transformations were used. Tumor-absorbed doses were calculated using the local deposition method, using the following formula:

$$
\mathrm{D}_{\text {tumor }}(\mathrm{Gy})=\frac{\mathrm{A}_{\text {tumor }}(\mathrm{GBq}) \cdot 50(\mathrm{~J} / \mathrm{GBq})}{\mathrm{V}_{\text {tumor }}(\mathrm{L}) * 1.03(\mathrm{~kg} / \mathrm{L})}
$$

Where $D_{\text {tumor }}$ is the tumor-absorbed dose in Gy, $A_{\text {tumor }}$ is the mean activity in the tumor VOI in GBq, 50 is the absorbed energy in joules from the decay of $1 \mathrm{GBq}$ of ${ }^{90} \mathrm{Y}, \mathrm{V}_{\text {tumor }}$ is the volume of the tumor VOI in L, and $1.03 \mathrm{~kg} / \mathrm{L}$ is the assumed density of liver tissue. 


\section{Dose-toxicity Evaluation}

Standard clinical protocol included clinical and laboratory evaluation at baseline, two weeks, one month, and three months after treatment. Laboratory markers collected for analysis included: serum albumin, total bilirubin, alkaline phosphatase (AP), gamma-glutamyltransferase (GGT), aspartate aminotransferase (AST), and alanine transaminase (ALT). All events were recorded using the Common Terminology Criteria for Adverse Events (CTCAE) version 5. Preexisting toxicities were excluded unless they exacerbated after treatment.

The whole-liver VOIs were manually delineated on low-dose CT and were subsequently expanded along the original contours by $10 \mathrm{~mm}$ to correct for errors due to motion and scanner resolution. All tumor VOIs that were $\geq 5.0 \mathrm{~mL}$ on baseline ${ }^{18} \mathrm{~F}$-FDG-PET were then subtracted from the original and the expanded whole-liver VOI. The healthy liver-absorbed doses were calculated using activity measured within the expanded VOI and the volume of the original VOI.

\section{Scanner Equipment, Acquisition, and Image Reconstructions}

PET-images were acquired on a Biograph mCT time-of-flight PET/CT scanner with TrueV (Siemens Medical Solutions USA, Inc.).

${ }^{90}$ Y-PET was acquired in two bed positions, with an acquisition time of $15 \mathrm{~min}$ per bed position. To reconstruct the images, an iterative algorithm (4 iterations, 21 subsets), including scatter correction, resolution recovery, and CT-based attenuation correction (40 mAs; 100-120 kV) was used. A Gaussian post-reconstruction filter of $5 \mathrm{~mm}(\mathrm{FWHM})$ was applied, and a $200 \times 200$ matrix, resulting in a pixel size of $4 \times 4 \times 3 \mathrm{~mm}^{3}$.

${ }^{18} \mathrm{~F}-\mathrm{FDG}$ PET imaging was performed 1 hour after injection of $2.0 \mathrm{MBq} / \mathrm{kg}{ }^{18} \mathrm{~F}-\mathrm{FDG}$. Images were reconstructed using a European association of nuclear medicine research Itd. (EARL) accredited protocol $(10,11)$.

\section{Statistical Analysis}

The relationship between tumor-absorbed dose (log-transformed) and response was analyzed using a linear mixed-effects regression model, to account for correlation of tumors within patients. Nested 
models were compared using Akaike's Information Criterion. A trend test was performed to test for an ordered relationship across the response categories; in this model response was used as a continuous variable. The dose-effect relationship was best explained using a random intercept per patient without random slopes. Analyses were adjusted for the following possible confounders: tumor volume, specific activity, dose heterogeneity (standard deviation of activity concentration within a tumor), primary tumor location, extrahepatic disease, and number of prior systemic treatment lines. A receiver operating characteristic (ROC) analysis, according for clustered data, was performed to determine the discriminatory power of tumor-absorbed dose in response estimation (10).

The strength of association between CTCAE toxicity grade and healthy liver-absorbed dose was assessed using linear regression models with CTCAE grade in categories as the dependent continuous variable and healthy liver-absorbed dose as the independent continuous variable. Simple linear regression models were used to assess the association between relative change in laboratory parameters (represented as $\Delta$ laboratory parameter) and healthy liver-absorbed dose. All toxicity analyses were adjusted for response to therapy (binary coded as response/non-response), mean tumor-absorbed dose (continuous variable), and hepatic reserve as possible confounders.

Overall survival was defined as the interval between radioembolization and death from any cause. Cox regression models were made using Firth's correction for small sample bias (11). Analyses were adjusted for age and presence of extrahepatic at baseline. Inspection of Schoenfeld residuals showed that the proportionality of the hazard assumption was not violated. Analyses were performed using R statistical software, version 3.6.2 for Windows. We report effect estimates with associated $95 \% \mathrm{Cls}$ and corresponding two-sided p-values. 


\section{RESULTS}

A total of 39 patients were treated with glass ${ }^{90} \mathrm{Y}$-radioembolization for CRLM, 31 of whom were included in this study (Table 1); $24 / 31$ (77\%) patients were included in the dose-response evaluation, and $28 / 31(90 \%)$ in the dose-toxicity evaluation (Fig. 2).

\section{Dose-response Evaluation}

A total of 85 tumors $>5 \mathrm{~mL}$ were identified in 24 patients and included for analysis. Median time from baseline ${ }^{18} \mathrm{~F}-\mathrm{FDG}-\mathrm{PET}$ to radioembolization was 31 days (range 8-70 days); Median time from radioembolization to follow-up FDG-PET was 89 days (range 59-112 days). The median delay between the reference time (date of dose was calibration) and microsphere injection was 4 days (range 1-11 days). Ten patients developed new lesions after radioembolization, three of whom had partial response based on the treated lesions. All three patients received unilobar or segmental radioembolization and developed new lesions in untreated parts of the liver.

Per-lesion Analysis. The median tumor-absorbed dose was 133 Gy (range 20-1001 Gy). The metabolic response in individual tumors at three-months follow-up was CR in 10 tumors (12\%), PR in 37 tumors (44\%), StD in 20 tumors (23\%), and PD in 18 tumors (21\%). The median tumor-absorbed dose per response category was, 196 Gy (98-1001 Gy) for CR, 177 Gy (59-551 Gy) for PR, 72 Gy (24-189 Gy) for StD, and 95 Gy (20-246 Gy) for PD (Fig. 3).

The mean tumor-absorbed dose was 94\% higher in CR compared to PD (95\% confidence-interval (Cl):47-157\%), 74\% higher in PR compared to PD (95\%Cl:42-112\%), and $2 \%$ higher for StD compared to PD (95\%Cl: -16-25\%), ptrend $<0.0001$ (Table 2).

Tumor-absorbed dose was found to be a good predictor of objective response based on ROC analysis with an area-under-the-curve of $0.88(95 \% \mathrm{Cl}$ : 0.79-0.98) (Fig. 4). A tumor-absorbed dose of $>139$ Gy predicted three-month metabolic response with the greatest accuracy ( $89 \%$ specificity, $77 \%$ sensitivity), while a tumor-absorbed dose of $>189$ Gy predicted response with $97 \%$ specificity and $45 \%$ sensitivity. 
Per-patient Analysis. There was a significant difference in mean tumor-absorbed dose between response categories on a per-patient basis. The geometric mean tumor-absorbed dose of all measured tumors (range) was 198 Gy in responders (CR+PR), 107 Gy in StD, and 94 Gy in PD (Fig. 5). The mean dose in responders was $58 \%$ higher than in patients with PD $(5-131 \%$, ptrend $=0.044)($ Table 2$)$.

\section{Dose-toxicity Analysis}

Healthy liver tissue-absorbed dose measurement was performed in 28 patients. Fourteen lesions $<5 \mathrm{~mL}$ (median $2.2 \mathrm{~mL}$ ) were found in 8 patients. These lesions could not be reliably subtracted from the healthy liver VOI. However, the total volumes of lesions included in the healthy liver were $\leq 0.6 \%$ of the VOI in all patients. The median whole-liver healthy liver-absorbed dose was $63 \mathrm{~Gy}$ (range: 24-113 Gy). Median follow-up was 91 days (range 14-110 days). Clinical data or $\geq 1$ laboratory datapoints were missing for two patients at one-month and four patients at three-months follow-up.

A total of 95 adverse events (AE) were recorded in 24/28 (86\%) patients and consisted of 47 laboratory and 48 clinical toxicities (Table 3). Three serious adverse events were observed (i.e., $\geq$ grade 3). One patient received an absorbed dose of $113 \mathrm{~Gy}$ to the healthy liver in a whole-liver treatment, using microspheres nine days post-calibration. They initially presented with mild symptoms of nausea, fatigue, and abdominal pain. Six weeks after treatment the patient developed ascites (grade 2). At three-months post-treatment, grade 3 GGT toxicity, grade 2 hyperbilirubinemia, as well as elevations in AP, AST, and ALT developed, confirming the diagnosis of radio-embolization induced liver disease. Symptomatic treatment was continued by the referring oncologist. The patient had a partial metabolic response to the treatment with a mean tumor-absorbed dose of $172 \mathrm{~Gy}$ and died 11 months after treatment.

The two remaining SAEs occurred in one patient, who experienced grade 3 GGT and AP toxicity as well as an elevation in bilirubin at three months after treatment, accompanied by mild abdominal pain, fatigue, and anorexia (all grade 1). The healthy liver-absorbed dose was $63 \mathrm{~Gy}$. A CT scan revealed a central biliary obstruction due to disease progression as the likely cause.

The highest healthy liver-absorbed dose without radio-embolization induced liver disease was 88 Gy. Most toxicities were mild, i.e., CTCAE grade 1 or 2 ( $n=71$ and $n=21$, respectively). The most 
frequently occurring clinical toxicities were fatigue, abdominal pain, and nausea, which were expected and generally resolved without intervention.

Linear regression analysis showed no significant relation between healthy liver-absorbed dose and any clinical toxicity grade (Supplemental Table 1). However, healthy liver-absorbed dose was related to laboratory toxicity grade (Supplemental Table 2 and Fig. 1) as well as relative changes in laboratory parameters (Supplemental Table 3).

\section{Survival}

The median overall survival (OS) in our sample was 13.2 months $(95 \% \mathrm{Cl} 8.4-18.9)$. The median OS of responders was significantly higher than non-responders, 16.9 months vs 8.7 months (Fig. 6). The hazard ratio for responders was $0.27(95 \% \mathrm{Cl}: 0.09-0.72, \mathrm{p}=0.0091)$. A mean tumor dose $\geq 189$ resulted in a higher OS, however, not statistically significant.

\section{DISCUSSION}

This study is the first to report a dose-response relationship in patients with CRLM treated with glass ${ }^{90} \mathrm{Y}$-microspheres. Our results show that higher tumor-absorbed doses result in better metabolic response at three-months follow-up, as well as improved survival. This relationship was demonstrated both on an individual tumor level and on a patient level. Furthermore, a tumor-absorbed dose >189 Gy was found to be a good predictor of metabolic response after three months.

Currently, dose-response relationships are best established in the treatment of HCC. In a prospective study in $35 \mathrm{HCC}$ patients, the median tumor-absorbed dose in responders (mRECIST 1.1) was 225 Gy compared to 83 Gy in non-responders (12). All tumors with a tumor-absorbed dose >200 Gy had a good response. A retrospective study on radiation segmentectomy in $33 \mathrm{HCC}$ patients found that $14 / 17$ tumors with complete pathological necrosis received an absorbed dose of $>190 \mathrm{~Gy}(p=0.03)$ to the treated segment (13). A recent consensus panel of experts therefore recommended a tumor-absorbed dose $>200$ Gy to achieve response (14). The findings of the present study appear within the same range, albeit in a different tumor type. 
Although a dose-toxicity relationship was not established, the results indicate that the treatment is well tolerated. Serious toxicity occurred in one whole-liver treatment that resulted in a very high healthy liver-absorbed dose of $110 \mathrm{~Gy}$. Apart from this outlier, treatments were well tolerated with doses up to 88 Gy. These results are in line with preliminary healthy liver-absorbed dose thresholds in HCC. A study on unilobar treatment of HCC identified bilirubin to be a significant risk factor for toxicity and determined safety thresholds based on its baseline value. A healthy liver-absorbed dose of 90 Gy poses a $15 \%$ risk of liver decompensation in patients with low baseline bilirubin (i.e., $<1.1 \mathrm{mg} / \mathrm{dL}$ ). The threshold for baseline bilirubin $>1.1 \mathrm{mg} / \mathrm{dL}$ was found to be $50 \mathrm{~Gy}(15)$. Other authors identified a combination of a healthy liverabsorbed dose $\geq 120$ Gy and $<30 \%$ non-irradiated liver volume to be a significant factor for toxicity (16). Interestingly, a 'perfused volume' healthy liver-absorbed dose of 120 Gy constitutes a whole-liver healthy liver-absorbed dose of around 84 Gy considering a $30 \%$ non-irradiated liver volume. Which is also comparable to the findings of the present study.

Our group conducted two similar studies in CRLM using resin ${ }^{90} \mathrm{Y}$-microspheres and ${ }^{166} \mathrm{Ho}$ microspheres $(5,6)$. These studies used the same methods for dosimetry (i.e., ${ }^{18}$ F-FDG-PET-based tumor delineation, post-treatment dosimetry, ${ }^{18} \mathrm{~F}$-FDG-PET-based response assessment). The study on resin $\mathrm{Y}^{90}$-microspheres estimated that a tumor-absorbed dose of 40-60 Gy was required to achieve a substantial metabolic tumor response (i.e., a TLG decrease of $\geq 50 \%$ ) at one-month follow-up. This is much lower than the absorbed dose thresholds found in the present study. The other study focusing on the dose-response relationship in ${ }^{166} \mathrm{Ho}$-radioembolization of CRLM found a mean tumor-absorbed dose of $173 \mathrm{~Gy}$ in responders (i.e., CR/PR), which is more in line with the $193 \mathrm{~Gy}$ found in responders in the present study (6). There are many factors that should be considered when comparing these studies, which include important differences in specific activity, number of injected microspheres, dosimetry technique, treated populations, etc. These differences between microspheres warrant further research.

The dose thresholds identified in this study were based on post-treatment 90Y-distribution instead of pre-treatment 99mTc-MAA distribution. In clinical practice, 99mTc-MAA distribution is used for determining the $90 \mathrm{Y}$-activity to be injected. We chose to study 90Y-distribution instead of 99mTc-MAA distribution since the known-mismatch between 90Y and 99mTc-MAA would add another factor diluting 
the observed dose-response relationship. The 99mTc-MAA to $90 \mathrm{Y}$-mismatch is unpredictable (17). Thus, caution is advised when using 90Y-based thresholds for 99mTc-MAA-based treatment planning.

The current study has several limitations. First, due to our limited sample size we were not able to establish absorbed dose thresholds for healthy liver tissue. This is a common problem in studies on radioembolization due to the low incidence of serious toxicity. Second, a follow-up period of three-months might be considered too short, however, in our experience, radioembolization-induced liver disease usually occurs within two months of treatment. Third, this cohort consisted mostly of heavily pretreated, chemorefractory, CRLM patients, therefore the results may not apply to patients in earlier lines of treatment. Additionally, there were large ranges in the timing of baseline and follow-up scans, limiting the precision of the reported results. The automated VOI delineation method used in this study decreases inter-operator variability compared to manual delineation and resulted in a more reproducible delineation result. Furthermore, the subsequent registration with ${ }^{90} \mathrm{Y}-\mathrm{PET}$ images based on individual tumor VOI produced superior tumor-absorbed dose measurements. However, our measurements will likely differ from those acquired with routinely used methods based on CT or MRI. Finally, metabolic response using TLG differs from the more widely used RECIST method. Nonetheless, metabolic response using TLG was used to combine volume changes and metabolic changes in one response metric $(18,19)$.

As we shift towards a personalized treatment approach in radioembolization, the demonstration of a dose-response relation in CRLM with glass ${ }^{90} \mathrm{Y}$-microspheres brings us a step closer towards this goal. Based on our data we recommend a tumor-absorbed dose of $>189$ Gy to achieve response, however this should be considered as a target and not as an absolute threshold for patient selection as sufficient response has been achieved with lower absorbed-doses.

\section{CONCLUSION}

A significant dose-response relationship for the treatment of CRLM patients with glass ${ }^{90} \mathrm{Y}$ microspheres was found. Patients that received higher tumor-absorbed doses showed better response rates. 


\section{DISCLOSURE}

Conflicts of interest:

$M L$ is a consultant for Boston Scientific and Terumo. MS and AB have served as speakers for SirTex, BTG and Terumo. AA has served as a speaker for BTG. MK has received research grants from SirTex. The Department of Radiology and Nuclear Medicine of the UMC Utrecht receives royalties from Quirem Medical. Medical and research support from Terumo, Quirem Medical, and BTG. No other potential conflicts of interest relevant to this article exist.

\section{KEY POINTS}

Question: Is there a relationship between dose and effect in glass ${ }^{90}$ Y-radioembolization of colorectal liver metastases?

Pertinent Findings: This retrospective cohort study demonstrated a significant dose-response relationship. A tumor-absorbed dose of >189 Gy predicted response with great specificity $(97 \%)$.

Implications for patient care: Our findings could be used to implement personalized dosimetry. 


\section{REFERENCES}

1. Reinders MTM, Mees E, Powerski MJ, et al. Radioembolisation in Europe: a survey amongst CIRSE members. Cardiovasc Intervent Radiol. 2018;41:1579-1589.

2. Garin E, Tselikas L, Guiu B, et al. Personalised versus standard dosimetry approach of selective internal radiation therapy in patients with locally advanced hepatocellular carcinoma (DOSISPHERE-01): a randomised, multicentre, open-label phase 2 trial. Lancet Gastroenterol Hepatol. 2020;1253:1-13.

3. Hermann A-L, Dieudonné A, Ronot M, et al. Relationship of tumor radiation-absorbed dose to survival and response in hepatocellular carcinoma treated with transarterial radioembolization with 90 Y in the SARAH Study. Radiology. 2020;296:673-684.

4. Garin E, Rolland Y, Laffont S, Edeline J. Clinical impact of 99mTc-MAA SPECT/CT-based dosimetry in the radioembolization of liver malignancies with 90Y-loaded microspheres. Eur J Nucl Med Mol Imaging. 2016;43:559-575.

5. van den Hoven AF, Rosenbaum CENM, Elias SG, et al. Insights into the dose-response relationship of radioembolization with resin 90Y-microspheres: a prospective cohort study in patients with colorectal cancer liver metastases. J Nucl Med. 2016;57:1014-9.

6. van Roekel C, Bastiaannet R, Smits MLJJ, et al. Dose-effect relationships of holmium-166 radioembolization in colorectal cancer. $\mathrm{J}$ Nucl Med. Epub ahead of print.

7. TheraSphere ${ }^{\circledR}$ yttrium-90 glass microspheres [Package Insert]. Biocompatibles UK Ltd, a BTG International group company. Rev. 14.

8. Bastiaannet R, van Roekel C, Smits MLJ, et al. first evidence for a dose-response relationship in patients treated with 166Ho radioembolization: a prospective study. J Nucl Med. 2020;61:608-612.

9. Joo Hyun O, Lodge MA, Wahl RL. Practical percist: a simplified guide to PET response criteria in solid tumors 1.0. Radiology. 2016;280:576-584. 
10. Obuchowski NA. Nonparametric analysis of clustered ROC curve data. Biometrics. 1997;53:567.

11. Heinze G, Dunkler D. Avoiding infinite estimates of time-dependent effects in small-sample survival studies. Stat Med. 2008;27:6455-6469.

12. Chan KT, Alessio AM, Johnson GE, et al. Prospective trial using internal pair-production positron emission tomography to establish the yttrium-90 radioembolization dose required for response of hepatocellular carcinoma. Int J Radiat Oncol. 2018;101:358-365.

13. Vouche M, Habib A, Ward TJ, et al. Unresectable solitary hepatocellular carcinoma not amenable to radiofrequency ablation: Multicenter radiology-pathology correlation and survival of radiation segmentectomy. Hepatology. 2014;60:192-201.

14. Salem R, Gabr A, Riaz A, et al. Institutional decision to adopt $\mathrm{Y} 90$ as primary treatment for hepatocellular carcinoma informed by a 1,000-patient 15-year experience. Hepatology. 2018;68:1429-1440.

15. Chiesa C, Mira M, Bhoori S, et al. Radioembolization of hepatocarcinoma with 90Y glass microspheres: treatment optimization using the dose-toxicity relationship. Eur $\mathrm{J} \mathrm{Nucl} \mathrm{Med} \mathrm{Mol}$ Imaging. Epub ahead of print.

16. Garin E, Rolland Y, Pracht M, et al. High impact of macroaggregated albumin-based tumour dose on response and overall survival in hepatocellular carcinoma patients treated with $90 \mathrm{Y}$-loaded glass microsphere radioembolization. Liver Int. 2017;37:101-110.

17. Wondergem M, Smits MLJ, Elschot M, et al. 99mTc-macroaggregated albumin poorly predicts the intrahepatic distribution of $90 \mathrm{Y}$ resin microspheres in hepatic radioembolization. $J$ Nucl Med. 2013;54:1294-301.

18. Shady W, Kishore S, Gavane S, et al. Metabolic tumor volume and total lesion glycolysis on FDGPET/CT can predict overall survival after 90Y radioembolization of colorectal liver metastases: A comparison with SUVmax, SUVpeak, and RECIST 1.0. Eur J Radiol. 2016;85:1224-1231. 
19. Woff E, Hendlisz A, Ameye L, et al. Validation of metabolically active tumor volume and total lesion glycolysis as 18 F-FDG PET/CT-derived prognostic biomarkers in chemorefractory metastatic colorectal cancer. J Nucl Med. 2019;60:178-184. 


\section{FIGURES WITH LEGENDS}
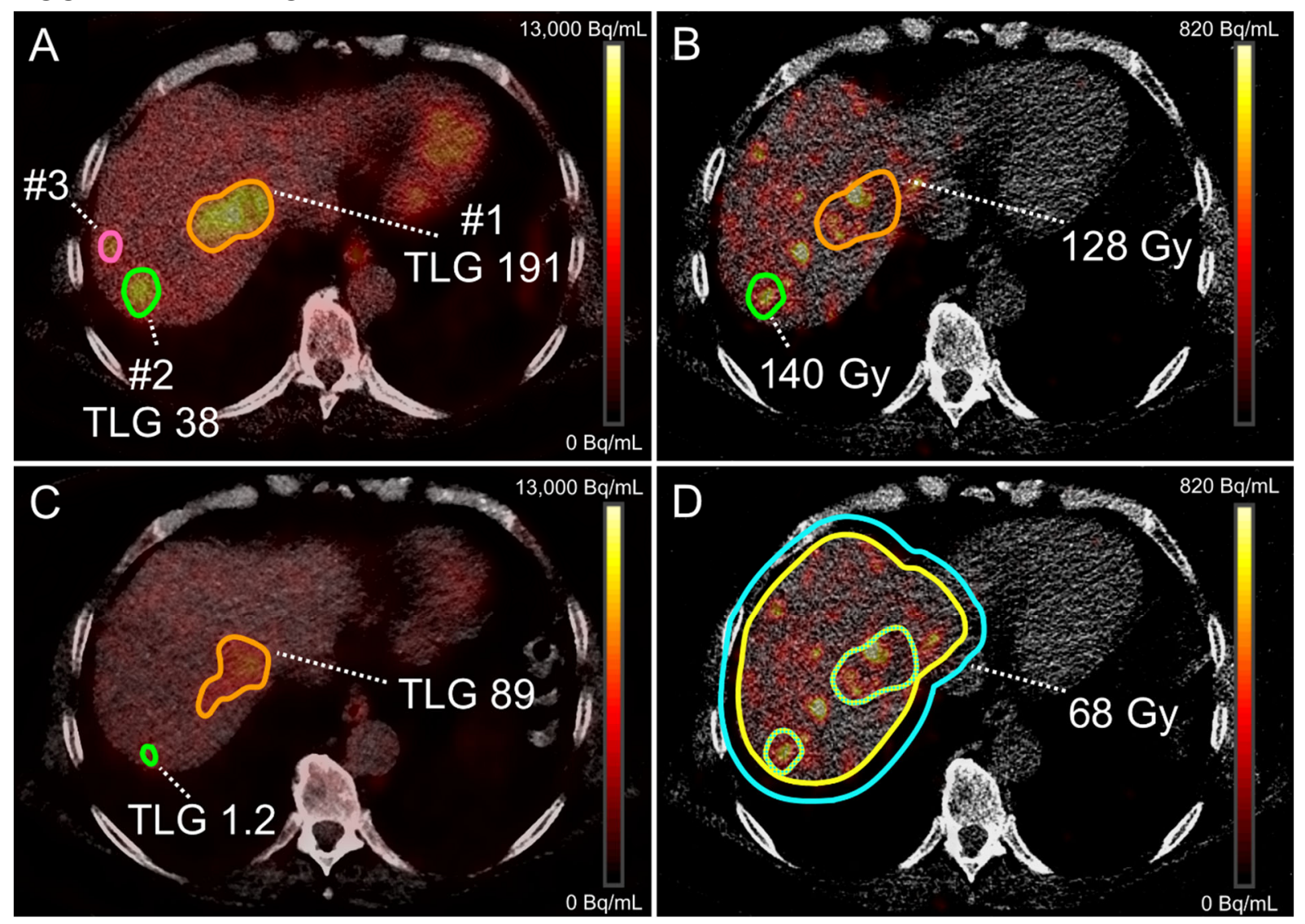

Figure 1. Example of absorbed dose and total lesion glycolysis (TLG) calculation. (A) A threshold-based mask on baseline ${ }^{18}$ F-FDG-PET to delineate three lesions and determine the baseline TLG, lesion 3 had a volume $<5 \mathrm{ml}$ and was excluded. (B) VOls were registered to post-treatment ${ }^{90} \mathrm{Y}-\mathrm{PET} / \mathrm{CT}$ to determine the individual tumor-absorbed dose. Notice the more heterogeneous dose distribution in lesion 1. (C) TLG measurement on the three-months follow-up ${ }^{18} \mathrm{~F}$-FDG-PET. Lesion 2 had a decrease in metabolic activity of $96 \%$ (PR), whereas lesion 1 only 53\% (PR). (D) The healthy liver-absorbed dose was measured on the post-treatment ${ }^{90} \mathrm{Y}-\mathrm{PET} / \mathrm{CT}$. The liver contour was manually delineated, this VOI was subsequently expanded by $10 \mathrm{~mm}$ in all directions to include all hepatic activity. A healthy liver VOI was achieved by subtracting all lesion VOls. 


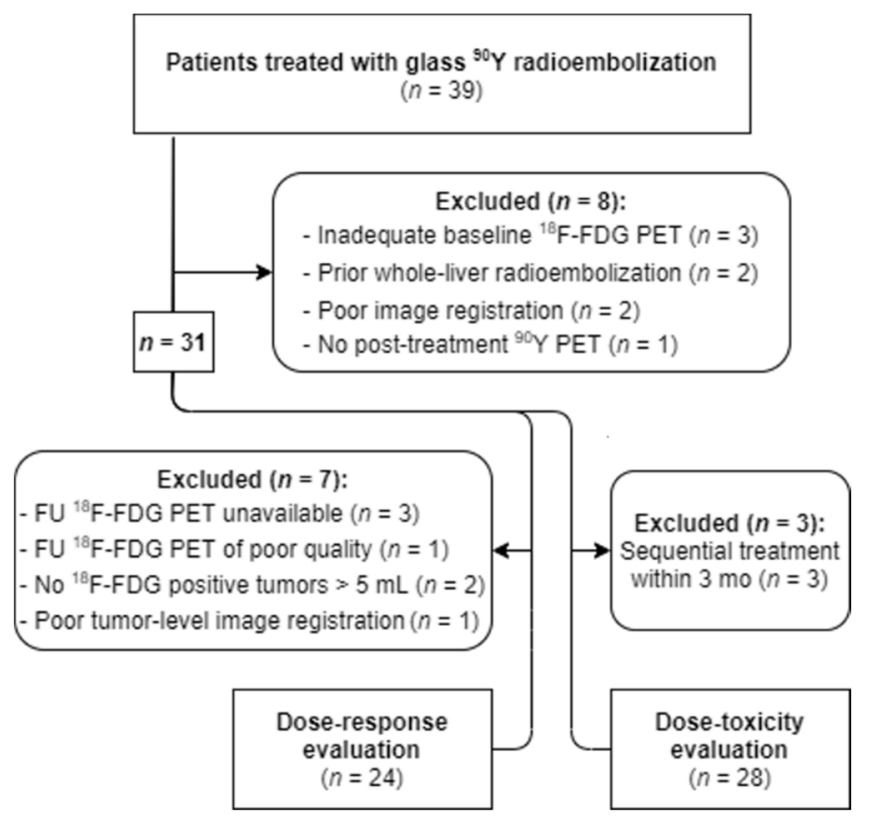

Figure 2. Flowchart of patient inclusion and exclusion. FU, follow-up. 


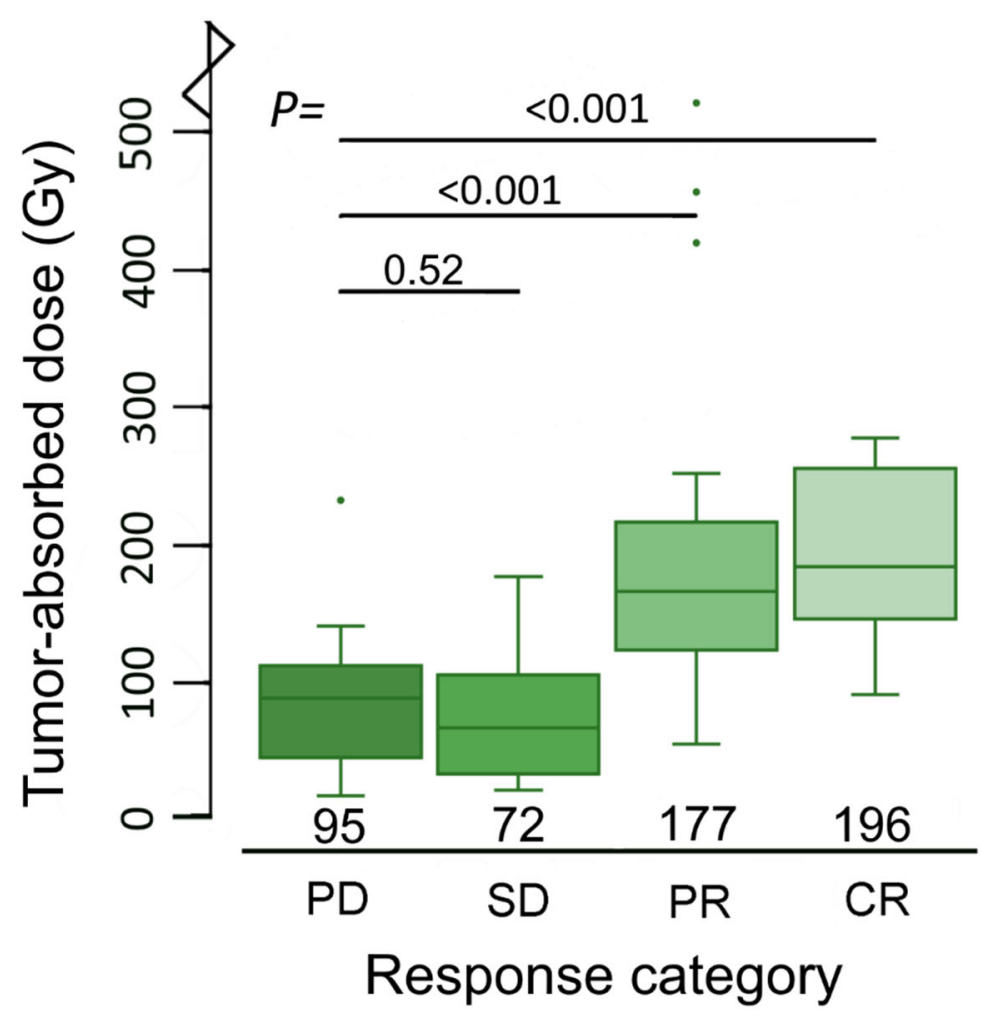

Figure 3. Boxplots demonstrating the relationship between tumor-absorbed dose on a tumor-level and metabolic response at three-months follow-up. Please note, one outlier in the complete metabolic response category (1001 Gy) was not depicted. 


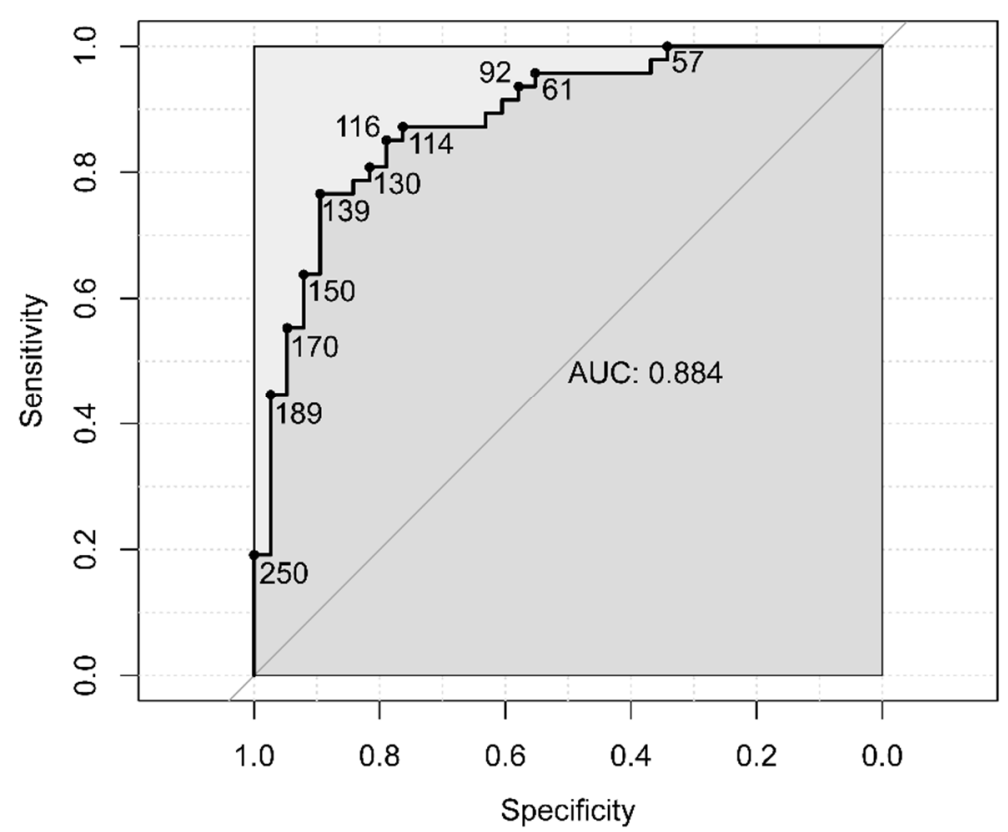

Figure 4. ROC-curve demonstrating the predictive value of tumor-absorbed dose for metabolic response $(C R+P R)$, on a tumor-level. The area under the curve (AUC) value is based on analysis of clustered data, whilst the ROC-curve is not. The ROC-curve is marked with the corresponding tumor-absorbed dose in Gray. 


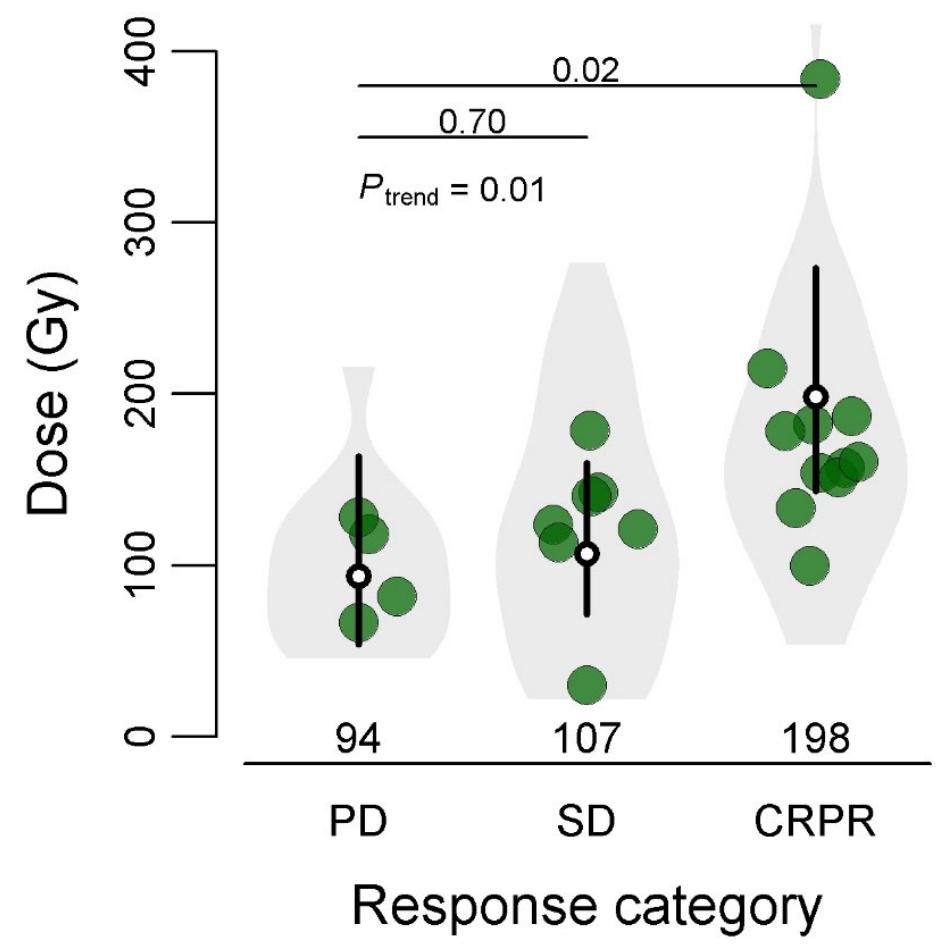

Figure 5. Relationship between tumor-absorbed dose on a patient level and metabolic tumor response at three-months follow-up. The white dots represent the mean tumor-absorbed dose per response category, 95\% confidence intervals are represented by the black lines. The large bullets depict the mean tumorabsorbed dose per patient. On a patient level only one patient was found with $\mathrm{CR}$, thus the categories $\mathrm{CR}$ and PR were taken together in the analysis. ${ }^{*}$ Geometric mean of tumor-absorbed dose. 


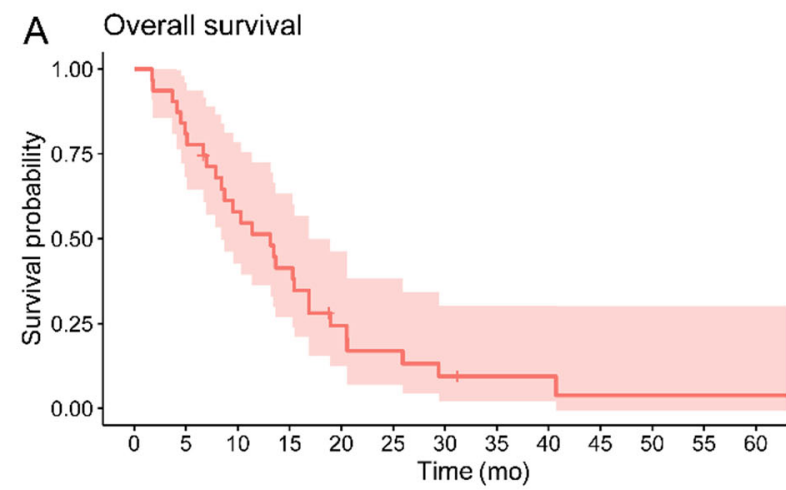

Number at risk

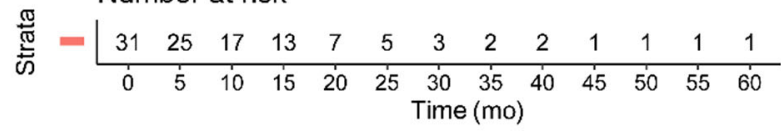

B Survival in responders vs non-responders

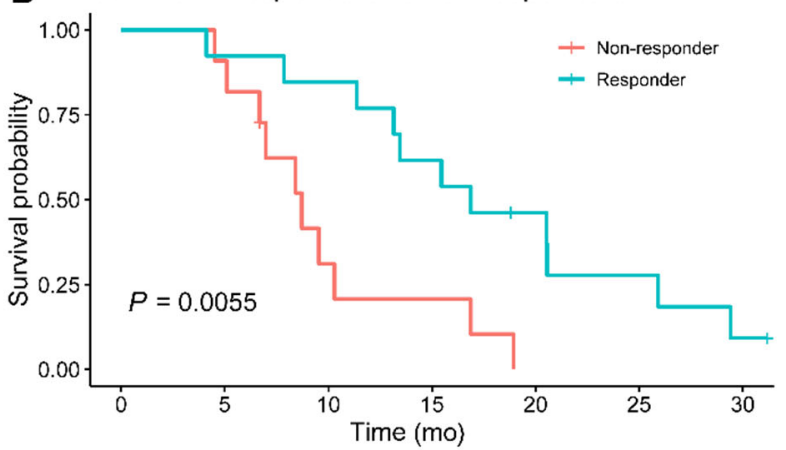

Number at risk

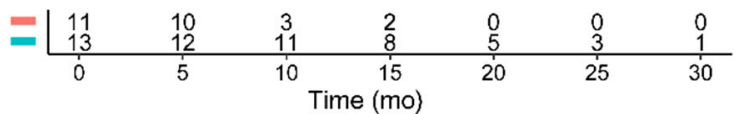

Figure 6. Kaplan-Meier curve of overall survival in all patients (A), and curves for patients with and without metabolic tumor response at three months. 


\section{TABLES}

Table 1. Baseline and treatment characteristics $(n=31)$

\begin{tabular}{|c|c|}
\hline Characteristic & $\mathbf{N}(\%)$ or median (range) \\
\hline \multicolumn{2}{|l|}{ Sex } \\
\hline Male & $25(81 \%)$ \\
\hline Female & $6(19 \%)$ \\
\hline Age (years) & $66(45-82)$ \\
\hline \multicolumn{2}{|l|}{ WHO performance score } \\
\hline 0 & $21(68 \%)$ \\
\hline 1 & $9(29 \%)$ \\
\hline 2 & $1(3 \%)$ \\
\hline \multicolumn{2}{|l|}{ Received prior therapy } \\
\hline Locoregional therapy* & $16(52 \%)$ \\
\hline Systemic treatment & $29(94 \%)$ \\
\hline Chemotherapy lines & $2(1-4)$ \\
\hline Bevacizumab & $16(52 \%)$ \\
\hline \multicolumn{2}{|l|}{ Extrahepatic disease at baseline } \\
\hline Yes & $12(39 \%)$ \\
\hline Lymph node & $8(26 \%)$ \\
\hline Lung & $4(13 \%)$ \\
\hline Other† & $2(6 \%)$ \\
\hline Liver volume (ml) & $1,890(821-3,030)$ \\
\hline Metabolic tumor volume (ml)‡ & $136(11-679)$ \\
\hline Tumors per patient & $3(1-8)$ \\
\hline Administered activity (MBq) & $2,925(1,193-5,994)$ \\
\hline Treated volume (ml) & $1,613(154-3,000)$ \\
\hline Treated fraction & $0.84(0.14-1.00)$ \\
\hline Average treated volume-absorbed dose (Gy) & $120(60-220)$ \\
\hline \multicolumn{2}{|l|}{ Radioembolization treatment } \\
\hline Whole-liver & $16(52 \%)$ \\
\hline Lobar§ & $15(48 \%)$ \\
\hline Synchronous metastasis & $13(42 \%)$ \\
\hline \multicolumn{2}{|l|}{ Primary tumor location } \\
\hline Left sided & $20(65 \%)$ \\
\hline Right sided & $3(10 \%)$ \\
\hline Rectum & $7(22 \%)$ \\
\hline Location unknown & $1(3 \%)$ \\
\hline \multicolumn{2}{|l|}{ Primary tumor status } \\
\hline In situ & $10(32 \%)$ \\
\hline Removed or chemo-irradiated & $21(68 \%)$ \\
\hline
\end{tabular}

${ }^{*}$ Includes ablative procedures (i.e. radiofrequency ablation $(n=4)$, microwave ablation $(n=1)$, hepatic surgery (i.e. metastasectomy $(n=8)$, segmentectomy $(n=1)$, hemi hepatectomy $(n=1))$, and portal vein embolization $(n=1)$. †One lung metastasis and one bone metastasis. ‡As per PERCIST. §Includes radiation lobectomy $(n=4)$ and radiation segmentectomy $(n=2)$ 
Table 2. Percentage change in mean absorbed dose (Gy) per response category $(95 \% \mathrm{Cl})$

\begin{tabular}{|c|c|c|c|c|c|}
\hline & $\begin{array}{l}\text { Progressive } \\
\text { disease }\end{array}$ & $\begin{array}{l}\text { Stable } \\
\text { disease }\end{array}$ & $\begin{array}{l}\text { Partial } \\
\text { response }\end{array}$ & $\begin{array}{l}\text { Complete } \\
\text { response }\end{array}$ & \\
\hline Patient-level & $\mathrm{n}=4$ & $n=7$ & $\mathrm{n}=12$ & $\mathrm{n}=1$ & \\
\hline Unadjusted & reference & $\begin{array}{l}13.6(-39- \\
112)\end{array}$ & $111(17-281)$ & - & $P_{\text {trend }}=0.0087$ \\
\hline Adjusted* $^{*}$ & reference & $29(-19-103)$ & $58(5-131)$ & & $P_{\text {trend }}=0.044$ \\
\hline Tumor-level & $n=18$ & $\mathrm{n}=20$ & $\mathrm{n}=37$ & $n=10$ & \\
\hline Unadjusted & reference & $-3.5(-29-31)$ & $90(40-161)$ & $159(62-286)$ & $P_{\text {trend }}<0.001$ \\
\hline Adjusted $†$ & reference & $2(-16-25)$ & $74(42-112)$ & $94(47-157)$ & $P_{\text {trend }}<0.001$ \\
\hline
\end{tabular}

${ }^{*}$ Adjusted for total tumor volume at baseline (per patient), specific activity and tumor dose heterogeneity, primary tumor location, extrahepatic disease, and number of prior systemic treatment lines. †Adjusted for tumor volume at baseline, specific activity, and tumor dose heterogeneity. 
Table 3. CTCAE grading of new clinical and laboratory toxicity per patient during three months after treatment

\section{CTCAE grade}

\begin{tabular}{|c|c|c|c|}
\hline Clinical toxicity & Grade 1 & Grade 2 & Grade 3 \\
\hline Abdominal pain & 9 & 1 & \\
\hline Nausea & 6 & 2 & \\
\hline Fatigue & 16 & 3 & \\
\hline Anorexia & 4 & 2 & \\
\hline Fever & 2 & & \\
\hline Constipation & & 1 & \\
\hline Ascites & 1 & 1 & \\
\hline Any clinical toxicity* & 15 & 7 & \\
\hline \multicolumn{4}{|l|}{ Laboratory toxicity } \\
\hline Albumin & 3 & 3 & \\
\hline Bilirubin & 1 & 2 & \\
\hline Alkaline phosphatase & 7 & 2 & 1 \\
\hline Gamma-glutamyltransferase & 4 & 2 & 2 \\
\hline Aspartate aminotransferase & 8 & 1 & \\
\hline Alanine transaminase & 10 & 1 & \\
\hline Any Lab toxicity* & 11 & 6 & 2 \\
\hline
\end{tabular}

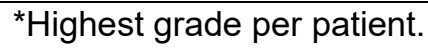




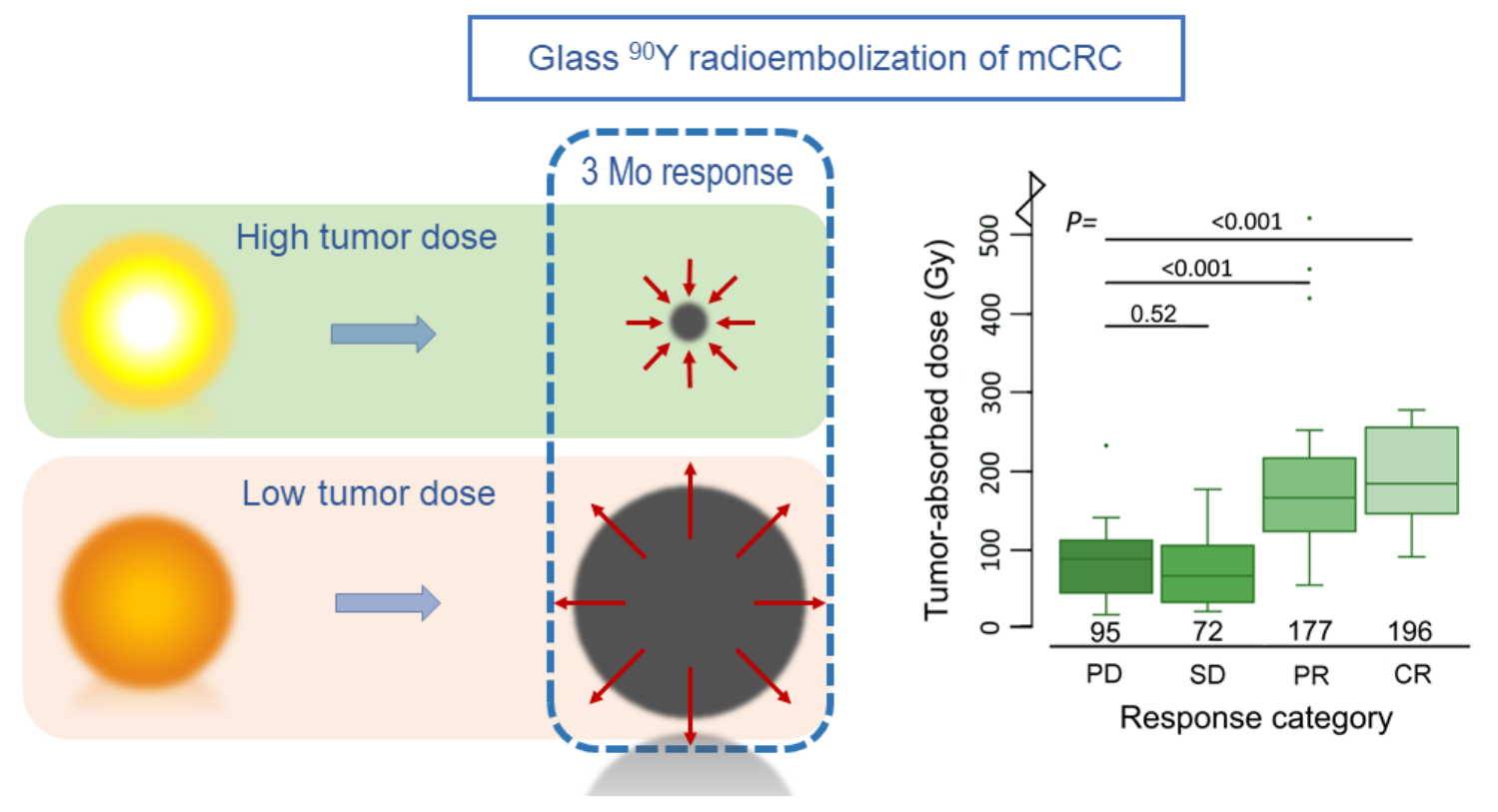




\section{Electronic supplement}

Supplemental table 1. Relation between healthy liver-absorbed dose (Gy) and clinical toxicity based on linear regression analyses with parenchymal dose as the dependent variable.

\begin{tabular}{llll}
\hline $\begin{array}{l}\text { Independent } \\
\text { variable }\end{array}$ & $\begin{array}{l}\text { Number of } \\
\text { patients with } \\
\text { toxicity }\end{array}$ & $\begin{array}{l}\text { CTCAE grade } 0-V \\
\text { Mean change in healthy liver- } \\
\text { absorbed dose (Gy) per step } \\
\text { increase in CTCAE grade } \\
\text { category (95\% Cl); p-value }\end{array}$ & Adjusted for treated fraction \\
\hline $\begin{array}{l}\text { Any variable, } \\
\text { highest grade }\end{array}$ & 22 & $-3.2(-14.3-7.9) ; 0.55$ & $-3.6(-14.5-7.2) ; 0.49$ \\
Abdominal pain & 10 & $1.3(-60.8-63.4) ; 0.96$ & $-10.5(-71.9-50.9) ; 0.70$ \\
Nausea & 8 & $-1.6(-15.2-11.9) ; 0.81$ & $-19.5(-58.8-19.8) ; 0.26$ \\
Fatigue & 19 & $-2.7(-32.0-26.5) ; 0.85$ & $-1.9(-27.4-23.6) ; 0.88$ \\
Anorexia & 6 & $-0.04(-27.1-27.0) ; 0.99$ & $4.3(-28.3-36.8) ; 0.71$ \\
\hline
\end{tabular}

The mean change indicates the average increase or decrease in healthy liver-absorbed dose per step increase in CTCAE grade toxicity. For example, for abdominal pain: a unit increase in toxicity results in an increase in average parenchymal dose of $1.3 \mathrm{~Gy}$. 
Supplemental table 2. Relation between healthy liver-absorbed dose (Gy) and cumulative laboratory toxicity over three months, based on linear regression analyses with healthy liverabsorbed dose as the dependent variable.

\begin{tabular}{llll}
\hline $\begin{array}{l}\text { Independent } \\
\text { variable }\end{array}$ & $\begin{array}{l}\text { Number of } \\
\text { patients with } \\
\text { toxicity }\end{array}$ & $\begin{array}{l}\text { CTCAE grade } 0-\mathrm{V} \\
\text { Mean change }(\mathbf{9 5 \%} \mathrm{CI}) ; \mathrm{p}-\mathrm{value} \\
\text { Unadjusted }\end{array}$ & $\begin{array}{l}\text { Adjusted for tumor dose and } \\
\text { response, and treated } \\
\text { fraction. }\end{array}$ \\
\hline Albumin & 5 & $-4.8(-17.6 ; 7.9) ; 0.44$ & $-4.7(-21.9 ; 12.4) ; 0.57$ \\
Bilirubin & 3 & $12.7(-2.2 ; 27.6) ; 0.091$ & $22.8(4.3 ; 41.4) ; 0.019$ \\
AP & 10 & $5.3(-5.3 ; 16.0) ; 0.31$ & $2.5(-8.3 ; 13.2), 0.64$ \\
GGT & 8 & $8.1(-0.44 ; 16.7) ; 0.062$ & $4.7(-4.3 ; 13.6) ; 0.28$ \\
ASAT & 9 & $7.1(-7.9 ; 22.1) ; 0.34$ & $0.5(-14.8 ; 15.8) ; 0.95$ \\
ALAT & 11 & $14.4(0.7 ; 28.1) ; 0.041$ & $9.7(-5.1 ; 24.6) ; 0.18$ \\
\hline
\end{tabular}

The mean change indicates the average increase or decrease in healthy liver-absorbed dose per unit increase in CTCAE grade toxicity. 

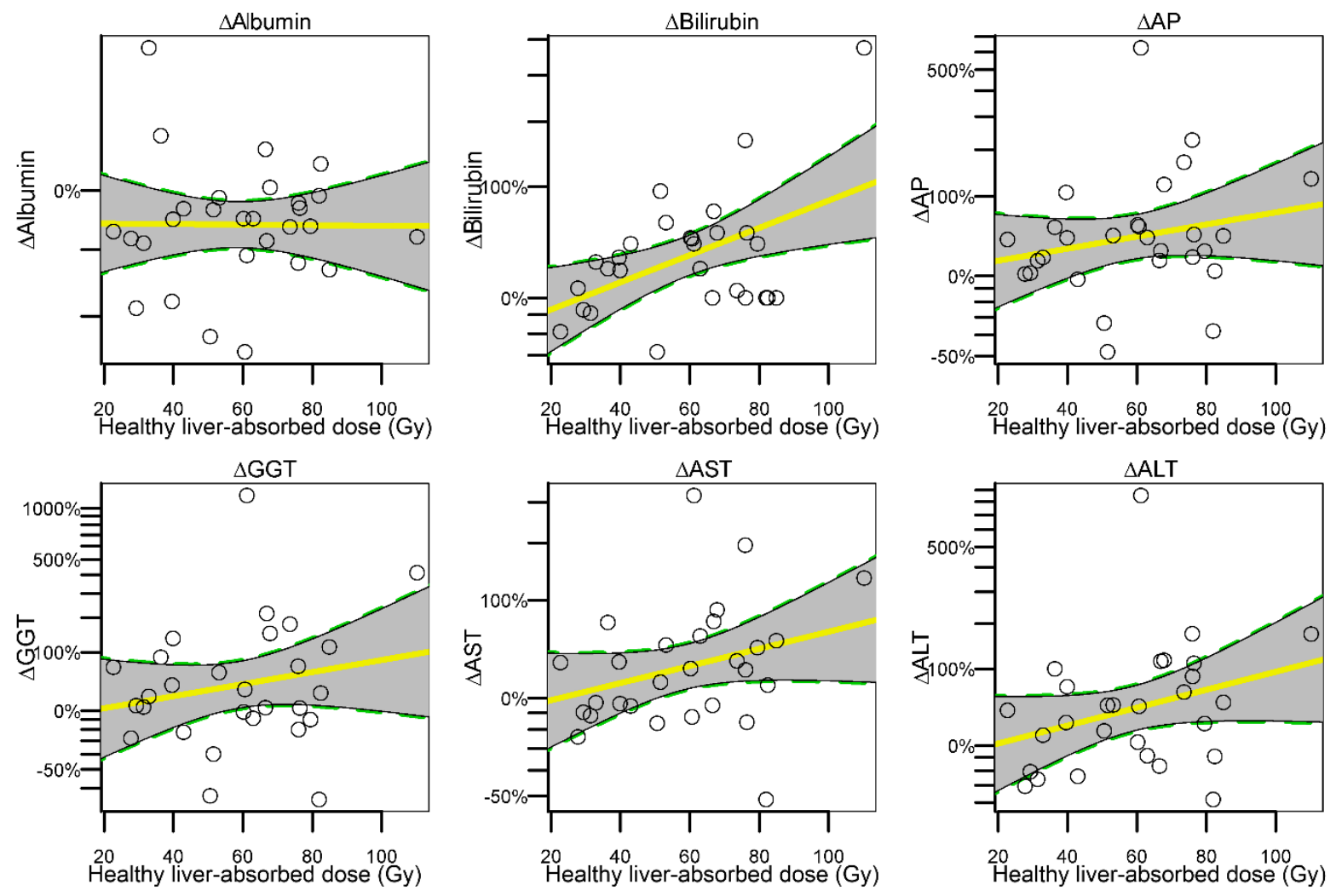

7 Supplemental figure 1. Graphical representation of the change in laboratory measurements vs. healthy 8 liver-absorbed dose in Gy with 95\% confidence interval 
Supplemental table 3. Relation between healthy liver-absorbed dose (Gy) and change in laboratory parameters over three months, based on linear regression analyses with healthy liver-absorbed dose (per $10 \mathrm{~Gy}$ ) as the independent variable

\begin{tabular}{lll}
\hline Dependent variable & $\begin{array}{l}\text { Mean percent change }(\mathbf{9 5 \%} \mathrm{Cl}) ; \mathbf{p} \text {-value } \\
\text { Unadjusted }\end{array}$ & $\begin{array}{l}\text { Adjusted for tumor dose and } \\
\text { response, and treated fraction. }\end{array}$ \\
\hline$\Delta$ Albumin & $-1.2 \%(-3.3 ; 1.1) ; 0.91$ & $-0.8 \%(-4.2 ; 2.6) ; 0.93$ \\
$\Delta$ Bilirubin & $8.1 \%(1.1 ; 15.5) ; 0.012$ & $11.9 \%(0.5 ; 23.3) ; 0.028$ \\
$\Delta$ AP & $3.9 \%(-6.2 ; 15.1) ; 0.34$ & $3.1 \%(-11.6 ; 20.2) ; 0.59$ \\
$\Delta$ GGT & $5.2 \%(-9.2 ; 21.8) ; 0.41$ & $6.0 \%(-14.5 ; 31.4) ; 0.51$ \\
$\Delta$ ASAT & $4.3 \%(-4.1 ; 13.5) ; 0.21$ & $0.5(-14.8 ; 15.8) ; 0.95$ \\
$\Delta$ ALAT & $6.1 \%(-4.7 ; 18.0) ; 0.20$ & $1.0 \%(-15.7 ; 21.0) ; 0.82$ \\
\hline
\end{tabular}

The mean change indicates the increase or decrease in average toxicity per 10 Gy increase in healthy liver-absorbed dose. 